\title{
NOTE ON HAHN'S THEOREM ON ORDERED ABELIAN GROUPS
}

\author{
A. H. CLIFFORD
}

In 1907, Hahn [2] showed that every (totally) ordered abelian group can be embedded in a lexicographically ordered, real function space. His proof occupies twenty-seven pages, not counting preliminaries, and may well be described as a transfinite marathon. For forty-five years, no one offered a simpler proof. But in 1952, Hausner and Wendel [3] gave a two-page proof of the same theorem for an ordered real vector space. In the present note, it is shown that the Hausner-Wendel proof applies equally well to the general case, with a few minor modifications, thus affording us an "accessible" proof of Hahn's fundamental theorem. Since this note is simply an appendix to [3], their definitions will not be repeated here.

(Added in proof. Not until after this paper was printed did the author become aware of the fine work of Paul F. Conrad [4], who not only simplifies the proof, but extends Hahn's Theorem to partially ordered abelian groups and to even more general systems. Conrad bases his proof on the intrinsic notion of a "decomposition" of the given group $G$, instead of the extrinsic notion of an order isomorphism of $G$ into an ordered function space. While basically equivalent, the two methods appear quite different, and perhaps it is just as well to have both in print.)

We begin by reducing the general case to that of an ordered rational vector space. It was shown by Baer $[1$, p. 768], that any abelian group $G$ having no element $\neq 0$ of finite order can be embedded in a minimal "complete" abelian group $V$, i.e. one having the property that, for any $v \in V$ and any positive integer $n$, there exists $x \in V$ such that $n x=v$. Baer takes for $V$ the set of all pairs $(x, n)$, with $x \in G$ and $n$ a positive integer, defining equality by

$$
(x, m)=(y, n) \text { if and only if } n x=m y,
$$

and addition by

$$
(x, m)+(y, n)=(n x+m y, m n) .
$$

$V$ may of course be regarded as a vector space over the field $Q$ of rational numbers.

Let now $G$ be any (totally) ordered abelian group. Since $G$ contains

Received by the editors March 18, 1954. 
no element $\neq 0$ of finite order, we may construct $V$ as above, and order $V$ by defining

$$
(x, m)>(y, n) \text { if and only if } n x>m y .
$$

$V$ becomes thereby an ordered vector space over the (ordered) field $Q$. Since every element of $V$ is a rational multiple of some element of $G$, every archimedean equivalence class of $V$ contains an element of $G$. Hence we may identify the set $T$ of archimedean equivalence classes of $V$ with that of $G$.

Let now $V$ be an ordered rational vector space. The development in $\$ 2$ of [3] holds, replacing "real" by "rational," up to Lemma 2.4. The latter we replace by the following considerations.

As in [3], we order the set $T$ of archimedean equivalence classes $[a]$ of $V$ "backwards": $[a]<[b]$ if $a \gg b$. For each $t \in T$, let $H_{t}\left(H_{t}^{\prime}\right)$ be the set of all elements $x$ of $V$ such that $[x] \geqq t([x]>t) . H_{t}$ and $H_{t}^{\prime}$ are convex (rational) subspaces of $V$. (A subspace $H$ of $V$ is convex if $h_{1}, h_{2} \in H$ and $h_{1}<x<h_{2}$ imply $x \in H$.) Since $H_{t}^{\prime}$ is complete in the sense of Baer, it is a direct summand of $H_{t}\left[3\right.$, p. 766], say $H_{t}=H_{t}^{\prime}$ $+K_{t}, H_{t}^{\prime} \cap K_{t}=(0)$. Since $H_{t}^{\prime}$ is a maximal proper convex subspace of $H_{t}, K_{t}$ is archimedean, and hence there exists an order-isomorphism $\phi$ of $K_{t}$ onto a rational subspace $R_{t}$ of the additive group $R$ of all real numbers. Without loss of generality we may assume that $R_{t}$ contains the real number 1 . Let $e_{t}$ be the element of $K_{t}$ such that $\phi\left(e_{t}\right)=1$. For any real number $\rho \in R_{t}$ we define $\rho e_{t}$ as that element $x$ of $K_{t}$ satisfying $\phi(x)=\rho$. This is consistent with the meaning of $r e_{t}$ for rational $r$.

(Actually, the choice of the (positive) element $e_{t}$ is quite arbitrary, and we may, if we like, pick $e_{t}$ in the original group $G$. We may avoid relying on the theorem of Baer cited by picking a rational basis of $R_{t}$, then picking representatives in the corresponding cosets of $H_{t} / H_{t}^{\prime}$, and finally by picking as representatives of the remaining cosets the correct rational linear combinations of these as dictated by the corresponding situation in $R_{t}$.)

We are now ready to give the substitute for Lemma 2.4.

Lemma. If $x$ is an element $\neq 0$ of $V$, there exists a uniquely determined number $\rho$ in $R_{t}$, where $t=[x]$, such that $x=\rho e_{t}$ or $x-\rho e_{t} \ll x$.

Proof. Since $x \in H_{t}, x$ is uniquely expressible as the sum $x=\rho e_{t}+x^{\prime}$ of an element $\rho e_{t}$ of $K_{t}\left(\rho \in R_{t}\right)$ and an element $x^{\prime}$ of $H_{t}^{\prime}$. If $x^{\prime} \neq 0$, then $x^{\prime} \ll x$ follows from the definition of $H_{t}$ and $H_{t}^{\prime}$, and the fact that $x \notin H_{t}^{\prime}$.

Turning now to the auxiliary Theorem 3.2 in [3], we make just two modifications. (1) The terms "linear mapping" and "subspace" are 
construed to mean relative to the rational field as operator domain. (2) The condition (iv) is extended to read:

$$
F\left(\rho e_{t}\right)=\rho f_{t} \text { for each } t \in T \text { and each } \rho \in R_{t} .
$$

In other words, our point of departure is the rational subspace $U_{0}$ of $V$ consisting of all finite sums of elements $\rho e_{t}$ of the subspaces $K_{t}$, and $F$ is defined on $U_{0}$ by

$$
F\left(\rho e_{t_{1}}+\cdots+\rho_{n} e_{t_{n}}\right)=\rho_{1} f_{t_{1}}+\cdots+\rho_{n} f_{t_{n}} .
$$

The subspace $V_{0}$ in Theorem 3.2 must contain $U_{0}$. Lemma 2.4 enters the proof of Theorem 3.2 in the first paragraph thereof, and the above lemma is seen to be an adequate substitute therefor. The remainder of the proof of Theorem 3.2, and that of the main Theorem 3.1, is readily seen to go through unscathed by the shift from "real" to "rational."

We remark that the proof also shows that $V$ can be embedded in the lexicographic product $\prod_{t \in T} R_{t}$ of the groups $R_{t}$. That this is not so for an arbitrary ordered abelian group $G$ is shown by the following example, due to Mr. Basil Gordon. Let $Q^{2}$ be the group of all vectors $(x, y)$ with rational coördinates $x, y$. Define $(x, y)>(0,0)$ to mean $x>0$, or $x=0$ and $y>0$. Let $G$ be the (ordered) subgroup of $Q^{2}$ generated by the vectors $\left(p_{n}^{-1}, n p_{n}^{-1}\right), n=1,2,3, \cdots$, where $p_{n}$ is the $n$th prime. $G$ contains just one proper nonzero convex subgroup $H$, namely the set of all $(0, y)$ with integral $y$, and it can be shown that $H$ is not a direct summand of $G$.

Theorem 3.2 is beautifully adapted to the proof of Hahn's completeness theorem $[2, \S 3]$. Hahn defines a complete ordered group to be an ordered group $G$ which does not admit a proper extension $H$ which is archimedean relative to $G$, in the sense that every archimedean equivalence class of $H$ contains an element of $G$. Hahn's completeness theorem asserts that an ordered group $G$ is complete if and only if it is order-isomorphic with the lexicographic function space $V_{T}$ defined on the set $T$ of archimedean equivalence classes of $G$. The "only if" is immediate from the main embedding theorem (Theorem 3.1), since $V_{T}$ is a relatively archimedean extension of $G$. To prove the "if," assume that $G \cong V_{T}$, and that $H$ is a relatively archimedean proper extension of $G$. Without loss of generality, we may assume that $H$ is a rational vector space. We apply Theorem 3.2 with $V=H$, $V_{0}=G, F$ the given order-isomorphism of $G$ onto $V_{T}$, and $x$ any element of $H$ not in $G$. By Theorem 3.2, there exists an extension of $F$ to the (rational) vector space $V_{1}$ spanned by $V_{0}$ and $x$, satisfying (i)-(v). Let $x^{\prime}$ be the image of $x$ in $V_{T}$ under this extension. Since 
$F(G)=V_{T}$, there exists an element $y$ of $G$ such that $F y=x^{\prime}$. Thus the distinct elements $x, y$ of $H$ are mapped by $F$ into the same element $x^{\prime}$ of $V_{T}$, contrary to condition (ii).

\title{
REFERENCES
}

1. Reinhold Baer, The subgroup of elements of finite order of an abelian group, Ann. of Math. vol. 37 (1936) pp. 766-781.

2. Hans Hahn, Über die nichtarchimedischen Grössensysteme, Sitz. der K. Akad. der Wiss., Math. Nat. Kl. vol. 116, Abt. IIa (1907) pp. 601-655.

3. M. Hausner and J. G. Wendel, Ordered vector spaces, Proc. Amer. Math. Soc. vol. 3 (1952) pp. 977-982.

4. Paul F. Conrad, Embedding theorems for abelian groups with valuations, Amer. J. Math. vol. 75 (1953) pp. 1-29. (Added in proof.)

The Johns Hopkins University

\section{A NOTE ON A RECENT RESULT IN SUMMABILITY THEORY}

\author{
C. F. MARTIN
}

In a recent paper ${ }^{1}$ by $A$. Mary Tropper, the following theorem is given:

THEOREM. In order that the regular ${ }^{2}$ normal ${ }^{3}$ matrix $A$ shall sum a bounded divergent sequence, it is sufficient that

(a) its unique reciprocal $B$ shall not be regular and

(b) there exists a normal matrix $Q$ with ${ }^{4}\|Q\|<\infty$ whose columns are all null sequences, such that the matrix $C=B Q$ has bounded columns and $\|C\|=\infty$.

The author points out that (a) is also a necessary condition, but does not prove the necessity of condition (b); the object of this note is to prove that condition (b) is necessary.

The proof given of the theorem quoted above holds if the $K_{r}$

Received by the editors January 5, 1954.

1 Proc. Amer. Math. Soc. vol. 4 (1953) pp. 671-677.

2 A regular matrix is one which satisfies $\lim _{n \rightarrow \infty} \sum_{k=1}^{\infty} a_{n, k} x_{k}=\lim _{k \rightarrow \infty} x_{k}$, whenever the latter limit exists.

${ }^{3}$ A normal matrix is a lower-semi matrix which has no zero elements in the leading diagonal. Such a matrix has a unique two-sided reciprocal; see e.g., R. G. Cooke, Infinite matrices and sequence spaces, Macmillan, 1950, p. 19.

${ }^{4}$ For an infinite matrix $M,\|M\| \equiv \sup _{n} \sum_{k}\left|m_{n, k}\right|$ is called the $K_{r}$ bound of the matrix: a matrix whose $K_{r}$ bound is finite is called a $K_{r}$ matrix (ibid. pp. 25, 29). 